\title{
The Effect of WeChat-assisted Problem-based Learning on the Critical Thinking Disposition of EFL Learners
}

\author{
https://doi.org/10.3991/ijet.v11i12.5927 \\ Xiao-Wei Ding \\ Beijing Information Science and Technology University, Beijing, China
}

\begin{abstract}
Literature suggests the potential of problembased learning (PBL) in critical thinking (CT) development, but empirical research on the effect of PBL on CT in foreign language pedagogy is limited. This study aims at a better understanding of the relationship between PBL and CT disposition of learners of English as a foreign language (EFL learners). A mixed research design was adopted in both an authentic in-class context and a WeChat-assisted after-class context, with an Adapted California Critical Thinking Disposition Inventory (CCTDI) as measurement tool of quantitative data, and with classroom observation notes and students' written comments on PBL experiences as qualitative data to supplement the quantitative analysis. The research findings confirmed that the PBL practice significantly improved EFL students' CT disposition in general, in Independent Inquiry step, and regarding all CT subscales. The study also revealed that the PBL practice eliminated significant gender difference in Group Negotiation, although there seemed a significant gender difference in Hypothesis Proposition after the PBL practice. Implications and limitations of this study, as well as suggestions for future research are also discussed accordingly.
\end{abstract}

Index Terms - critical thinking; problem-based learning; WeChat-assisted; EFL learner; CCTDI

\section{INTRODUCTION}

The 21 st century, with its features of globalization and digitalization, does not demand the teaching of soon-to-be obsolete facts, but the fostering of critical thinking (CT) at all levels of education [1]. The Foundation for Critical Thinking defines CT as a mode of thinking-about any subject, content, or problem-in which the thinker improves the quality of his or her thinking by skillfully taking charge of the structures inherent in thinking and imposing intellectual standards upon them [2]. CT is argued to be improved (rather than generated) through practice, and among diverse approaches, either direct or indirect, to CT development, problem-based learning (PBL) is widely believed to potentially promote CT [3].

PBL has been stated as a total approach to education [4] that develops CT, problem-solving skills and teamwork skills of learners by encouraging students to become selfdirected learners and to work cooperatively in small groups to seek solutions to problems [5]. Practitioners tend to refer to the procedures of classical or improved models of PBL [6] before adapting, designing and implementing their own PBL practice according to the features of the specific discipline and learners, but generally, 5 steps of PBL could be recognized in the current literature, namely "Problem Definition", "Hypothesis Proposition", "Group Negotiation", "Independent Inquiry", and "Selfreflection".

Quite a number of studies have examined the practice of PBL to enhance CT, but findings are mixed. A systematic review in medical and nursing science [7] included studies between 1999 and 2006 and demonstrated that theoretically the use of PBL may promote CT, but the available evidence did not provide supportive evidence. However, another review [8] indicated a positive relationship between PBL and improved CT in nursing students. Recently, researchers are increasingly interested in the relationship between PBL and CT in the Internet- or Hightech context. For example, a study on the development of CT through online PBL in graduate education courses revealed that online $\mathrm{PBL}$ promotes the development of $\mathrm{CT}$, and that through online PBL, participants developed a habit of CT and changed their teaching into more critical pedagogical practices [9]. However, empirical studies on the impact of PBL on CT in language teaching are relatively limited, none of which involves learning assisted by social interaction applications. What's more, no research has ever attempted to explore students' CT according to separate procedures of PBL.

The current study aims at exploring into this untouched research gap, and contributing to a better understanding of the relationship between PBL and CT disposition of EFL learners (the insufficiently-investigated community) in both an intact in-class context and a WeChat-assisted after-class context. The necessity of the assistance of WeChat is that the after-class group collaborations and self-directed inquiry of EFL learners must be realized and mediated via an internet-based social interaction application which overcomes the limitations of time and space. Since Chinese netizens have little access to the foreign social applications such as facebook messenger, skype messenger, google messenger, etc., while WeChat is the most popular instant social communication application in China, the current study chose WeChat as the communicative instrument assisting the after-class PBL practice. What's more, WeChat is especially welcomed by college students, because it can be conveniently installed either on computers or smart phones, and college students can instantly send and receive messages in forms of texts, photos, audio and video clips thanks to the campus intranet and Wi-Fi. Therefore, WeChat is the best choice of the instant communicative instrument for the current research, particularly because of its powerful communication function and social attribute facilitate interactions among users in China [10]. 
The current study adapted California Critical Thinking Disposition Inventory (CCTDI) into a Likert scale applied to EFL learners, which distinguishes the subscales of CT disposition and PBL steps, and serves as a pre-test and post-test of a 9-week PBL practice in a College English course for undergraduate students. The key research questions are:

1. What impact does the PBL practice have on the critical thinking disposition of EFL students in general?

2. Which subscales of the critical thinking disposition of EFL students are significantly affected by the PBL practice?

3. Which PBL procedures significantly influenced the critical thinking disposition of EFL students?

4. Is there any gender difference in the critical thinking disposition of EFL students in general, in subscales of critical thinking, and in PBL procedures?

\section{Methodology}

\section{A. Participants}

12 classes were randomly chosen from the total number of 52 classes of the course of "College English" at a university in Beijing, China. College English is a required course for undergraduate non-English majors in their freshman and sophomore year. There are typically up to 35 students in one College English class, who take this class twice a week, 2 hours each time, over 18 weeks. On the first day of school, 342 students registered in these 12 classes, 221 males and 121 females, who participated in the pre-test. Two weeks later, 3 male students were transferred from other schools, who were not included in the pre-test but participated the post-test. All of the student participants were freshmen majoring in information technology and mechanical engineering, and they all had started to learn English in their elementary school. 4 instructors, including the researcher herself, were in charge of the WeChat-assisted PBL College English course, and formed a community of practice, collectively worked out their PBL materials, and undertook their teaching based on a core teaching plan and on the same textbook with the same content and themes.

\section{B. Instrument}

The California Critical Thinking Disposition Inventory (CCTDI) was adapted to measure the students' critical thinking. Originally the CCTDI is a 75-item Likert scale tool with 7 subscales, namely Truthseeking (intellectual honesty, the desire to seek the best knowledge objectively even if the findings do not support one's self-interest or preconceived opinions), Open-mindedness (tolerance of new ideas and divergent views), Analyticity (alertness to the need to use reason and evidence), Systematicity (inclination to be organized, orderly, focused and diligent), Critical Thinking Self-confidence (trust in one's own reasoning and ability to guide others to make rational decisions), Inquisitiveness (intellectual curiosity and the desire for learning) and Cognitive Maturity (inclination to see the complexity in problems and the desire to be prudent in making decisions) [11]. In the current study, the CCTDI was adapted into 20 -item 5-point Likert scale with the same 7 subscales, based on the 5 steps of PBL, namely Problem Definition, Hypothesis Proposition, Independent Inquiry, Group Negotiation, and Self-reflection (see Table I).

\section{Intervention}

The students of all the 12 classes had traditional College English course during the first 9 weeks, and then participated in the PBL College English practice in the following 9 weeks. There was no control group in the research design, because the researcher believes that every student has the right of experiencing the PBL College English class based on the ethnic consideration in educational research, so the current study was conducted in a natural teaching context with a pretest-post-test design. The students in each class were divided into 4 to 5 groups, each group consisting of 7 or 9 students. The grouping was based on the students' free will. Usually, students chose their own group members based on adjacency or familiarity among them. Gender and abilities of the students were not necessary factors considered in the grouping process. The teacher in each class was the tutor of each PBL tutorial group in that class. The process of the PBL practice was as follows:

(1) Problem Definition: The problems were embedded in news reports, video clips, or reading materials, and were presented in English. The teacher participants made a deliberate effort to guide the students by offering insights on the problem's relevance, and the value that could be created by solving it. However, no specific explanation was provided as to how to do so. The student groups were guided to probe into the PBL materials, to clarify what happened in the materials, to define one problem which was related to the theme in that unit, and which was in the common interest of the whole group. For example, one of the reading materials is entitled "Fable of the Lazy Teenager" which is authored by Benjamin Stein. The fable is about a story of seven generations of "Kevin Hanley", all at the age of 17. The teacher participants encouraged each group to scan through this article, and to figure out a "problem", either linguistic or cultural, which puzzled or interested the whole group most. According to the classroom observation notes and written comments, some groups proposed linguistic problems, such as "In the sentence 'To drive this message home to such young Americans, I have a humble suggestion', we find 'drive home' here difficult to understand." or "What is the meaning of the citation 'A man without the proper use of the intellectual faculties of a man is, if possible, more contemptible than even a coward'?" Some groups put forward problems regarding discourse and comprehension issues, such as "Why does the author tell a true story in his own life before telling us the fable?" or "Why did Kevin 1990 change his mind after waking up from his dreams?" Other groups focused on cultural problems, such as "The author bought file folders in a drugstore, but we only buy medicines in a drugstore. Why are American drugstores different from their Chinese counterparts?" or "Are American teenagers still the same as Kevin 1990 in the fable?" The teacher walked around to encourage diverse thoughts and proposals within each group, and to discourage similar or shallow group problems within the class.

(2) Hypothesis Proposition: The PBL tutorial groups were guided by the tutor to discuss the defined problem based only on their prior experiences, on the English knowledge that they had just learned in that unit, or even on their knowledge of the world. The aim of such initial discussions is to construct a tentative hypothesis, in the form of needed information, explaining the phenomena or events described in the PBL materials. Taking one of the 
groups for example. Their group problem was "Why did Kevin 1990 change his mind after waking up from his dreams". After their initial tutorial group discussion, they reached an agreement that they needed to find out more information in the reading material so as to explain their group problem. Their hypothesis was as following: since the two key words in this problem are "change" and "dreams", what happened in his dreams might be critical to his change; among all the happenings in his dreams, there should be some specific factors resulted in his change; to identify the important factors, they must understand what his change at last was. Therefore, they presumed that it was necessary for them to search for the information they needed "from dreams to the change" and "from the change to dreams", that is, forward and backward when re-reading the material.

(3) Independent Inquiry: Based on the initial hypothesis, tutorial groups were further guided to undertake group discussions through WeChat after class, clarifying and formulating their group problem into several subproblems as the language learning content of their selfdirected study. Since the student in this university have flexible access to WeChat on their smart phones or computers, and the campus intranet and $\mathrm{Wi}-\mathrm{Fi}$ are free, WeChat as a research instrument in this research successfully overcame the spatial and temporal limitations of face-to-face or computer-assisted online interactions. Therefore, it was really convenient for the student participants to efficiently negotiate and assign group tasks (e.g. the sub-problems) within the tutorial groups. In fact, they communicated instantly with each other in not only the class group chat, but also their tutorial group chat or private chats with group members and their tutor, without having to be physically assembled at a pre-arranged time and in an all-agreed location. Thanks to the facilitation of WeChat, gradually each student chose one sub-problem (for example, the sub-problems of the above-mentioned group included "The experiences of Kevins", "The impact of these experiences on Kevin 1990", "The factors resulting in the change of Kevin 1990", "The change of Kevin 1990", etc.), and independently collected and selected new information (new knowledge of English) to explain or solve the sub-problem he or she was responsible to deal with after class. They then posted digital handouts on WeChat group chats for their peers, and proposed challenging questions for the following group discussions. The tutor mediated and supervised the group discussions and individual inquiries in class chat, group chat, and private chats, the social interactional functions offered by WeChat.

(4) Group Negotiation: During and after the independent inquiry, tutorial groups kept sharing and evaluating what they had learned, negotiating and communicating with each other any time when they were troubled relying on the same conversational functions of WeChat. Since it is assumed that this kind of elaboration helps students in the long-term retention of the EFL learning, the tutor encouraged the students to think broadly and deeply and gave comments on each handout, so as to help the students probe their new knowledge critically. Eventually, the tutorial groups reached a final solution to their group problem by integrating the valid and useful knowledge from each handout. As for the above-mentioned group, they finally reached a conclusion explaining their group problem that the attitude towards the education changed
Kevin 1990 from a lazy teenager to a serious student, because different attitudes towards the education resulted in different personal experiences as well as different directions of social development of a nation.

(5) Self-reflection: Finally, the tutorial groups returned to the class and reported in English what they had learned regarding their group problem, after they were guided to engage in self-evaluation, peer evaluation, and reflection on what they had learned and how they had learned it. The tutor helped them critically reflect on and evaluate their PBL experience through face-to-face conversations and cross-examinations.

\section{Data Collection}

The data were collected by means of a pre-test, a posttest, the researcher's regular classroom observation notes during the PBL practice, and the written comments of PBL experience from the student participants at the end of the PBL practice. At the beginning and the end of the PBL practice, student participants were asked to complete the Adapted CCTDI in class, and to voluntarily hand their survey paper in to the researcher after 15-20 minutes. The students were required to choose the number (1 to 5) best representing the degree of his or her agreement or disagreement to each statement in the Adapted CCTDI according to his or her previous English learning experience (as for the pre-test) or the PBL experience in English class (as for the post-test). In the pre-test, 334 survey papers were collected, 316 valid, 210 from males and 106 from females. In the post-test, 329 survey papers were voluntarily handed in, 312 valid, 222 from males and 90 from females. Those survey papers with one or more than one items left unanswered, or with identical answers to all items were regarded invalid and discarded.

\section{E. Data Analysis}

In order to answer the research questions, a descriptive analysis and an independent sample T-test of pre-test and post-test data were respectively conducted in SPSS 19.0 in order to see the whole picture of CT disposition of student participants before and after the PBL practice. In addition, the results of a cross-test paired-sample T-test were also checked and interpreted to find any significant changes of CT skills reported by student participants. Written comments were manually analyzed via the theme-related text analysis, the results of which were used to supplement the quantitative data and to help better understand what the students reckoned their CT disposition in a PBL context.

However, it should be noticed that although SPSS is a common tool for hypothesis testing, its use does not guarantee proper data analysis. In the case of the current study, since the tests were anonymous, and the students handed in their survey paper on the basis of voluntariness, it was impossible to exclude from the pre-test data or post-test data the answers of the students who participated in only one of the tests. What's more, another consequence of the "voluntariness" was the percentage of students (especially female) who did not complete the post-test, which potentially impaired the validity of the research results. These biases in the data are regarded as systematic errors, which should be taken into consideration when understanding the final results, because no statistical tools (such as SPSS) can compensate for lack of proper treatment of systematic errors. 


\section{RESULTS AND DISCUSSION}

\section{A. Pre-test \& Post-test}

In order to probe into the general situation of the CT disposition of the English learners in this study, a descriptive analysis of the pre-test and post-test data was performed (see Table I).

Table I shows that before the PBL practice, the student participants were weakest in Inquisitiveness, Cognitive Maturity and Open-mindedness subscale, as well as Problem Definition and Hypothesis Proposition procedure. However, the students reported quite optimistically concerning Critical Thinking Self-confidence, Truth-seeking, and Analyticity subscale, and Group Negotiation and Selfreflection procedure compared with other subscales and procedures. Their self-reported confidence in CT, in truthseeking and analytic ability, and in group negotiation and self-reflection skills were indeed encouraging for the researcher and other 3 teacher participants, because these results seemed supportive that the students seemed fairly ready for the critical and reflective group collaboration in the following PBL practice.

After the 9-week PBL practice, the picture of student participants' CT disposition was excitingly changed. When it comes to CCTDI subscales, the student participants still self-reported that they were weakest in Cognitive Maturity, Open-mindedness and Inquisitiveness, only with Inquisitiveness from top weakest to the third weakest. As far as PBL procedures were concerned, the CT of student participants in steps of Problem Definition and Hypothesis Proposition was somewhat improved, but the students still saw their CT in these two steps as the weakest, and that in Group Negotiation as the strongest. However, the most exciting result was that the mean value of Independent Inquiry of the students after the PBL practice was higher than that of Self-reflection, reversing the order of these two in the pre-test. It is reasonable to draw a conclusion that the PBL practice seemed to successfully improve the CT disposition of the student participants in terms of independent inquiry, and to maintain their CT in terms of group collaboration.

In order to answer the last research question, the researcher also tested gender difference(s) of the pre-test and post-test data at the significant level of 0.05 (see Table II). It could be found that in the pre-test, male students were generally less optimistic than female students about their CT subscales and critical skills that would be required in different steps of PBL, except for Systematicity. In particular, the mean value of female students regarding Group Negotiation was significantly higher than that of male students. As a result, it could be predicted that the male students might be faced up to more challenges in group discussion. This prediction was verified by the performance of male students at the beginning of the PBL practice: they were less good at listening, less willing to compromise in face of divergences, and more likely to impose their own opinions on others.

However, after the 9-week PBL practice, the previous significant gender difference in terms of Group Negotiation in the pre-test disappeared, which means the PBL experience of male students dramatically improved their CT in group collaboration up to a level statistically similar to that of female students. Instead, there emerged a significant gender difference in terms of Hypothesis Proposition, and male students scored higher than female ones.
The text analysis of students' written comments revealed that boy students generally underwent more intensive critical thinking experiences during the step of Hypothesis Proposition, because they are more "active" and "willing" to "freely express different opinions", and are "not as discouraged and frustrated as female students are when rejected and criticized", and therefore it is more likely for male students to work out bolder and more original assumptions. In contrast, girl students are much more "sensitive to the interpersonal relationship and to others' opinion" about themselves. With "thin face", they are more inclined to "maintain harmonious atmosphere" in interpersonal interactions, and are more reluctant to propose a unique and wild hypothesis, in order to "avoid the risk of losing face". In fact, the results of written comment analysis was in consistence with the classroom observation: girls reached in-group consensus faster than boys, because they made every effort to propose carefully after thorough consideration, and seldom opposed to others' proposals. Instead, they tended to supplement the ideas of each other, and expressed their opinion in a "safe" way. This gender difference in CT, however, was not likely to be due to the $\mathrm{CT}$ ability of female students, because there was no significant differences in any subscales of CCTDI. Rather, the gender socialization of females which leads them to be obedient and respectful to others, and which is rooted in the long history of oriental culture, might result in this significant gender difference.

In short, as to the last research question (Is there any gender difference in the critical thinking disposition of EFL students in general, in subscales of critical thinking, and in PBL procedures?), the answer, according to the above analysis, is that there was significant gender difference in the CT disposition of EFL students in Group Negotiation before the PBL practice, and in Hypothesis Proposition after the students experienced the 9-week PBL practice. This result had more to do with gender characteristics, rather than with CT itself, because there were no significant differences in the students' CT disposition in general, nor in CT subscales.

\section{B. Pre-test vs. Post-test}

In order to answer the 3 research questions, pairedsample T-tests were performed in SPSS 19.0 regarding all samples, in general first, and then in categories of PBL procedure and CCTDI subscale (see Table III). Table III shows very significant differences $(\mathrm{p}<0.01)$, or significant positive changes, in respect of the whole sample body, PBL procedures, and CCTDI subscales. In other words, the PBL practice of this study significantly improved (1) the EFL learners' CT disposition in general, which serves as a positive answer to the 1 st research question; (2) the 7 CT subscales of the EFL learners, which answers the 2nd research question; (3) and the CT disposition of the EFL students in 5 steps of PBL, that is, all of the PBL procedures significantly influenced the critical thinking disposition of EFL students.

The overwhelmingly optimistic results were in agreement with the positive findings of the relationship between PBL and CT in non-medical disciplines in the existing literature, and suggested that PBL has a statistically significant impact on not only students' CT in general and group task performance, but also their CT subscales and independent inquiry performance. As a result, the significant improvement of all CT subscales in the current study 
PAPER

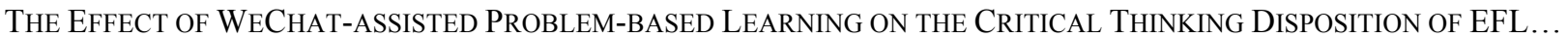

TABLE I.

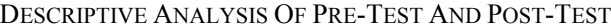

\begin{tabular}{|c|c|c|c|c|c|c|c|c|}
\hline & \multirow[t]{2}{*}{ Item Category } & \multirow[t]{2}{*}{ Item No. } & \multicolumn{3}{|c|}{ Pre-test } & \multicolumn{3}{|c|}{ Post-test } \\
\hline & & & $N$ & Mean & $S D$ & $N$ & Mean & $S D$ \\
\hline \multirow{7}{*}{ 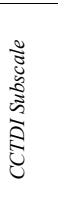 } & Inquisitiveness & 1,2 & 316 & 3.02 & 0.99 & 312 & 3.60 & 0.96 \\
\hline & Open-mindedness & $3,7,12$ & 316 & 3.11 & 0.79 & 312 & 3.59 & 0.77 \\
\hline & Cognitive Maturity & $4,16,18$ & 316 & 3.07 & 0.78 & 312 & 3.44 & 0.76 \\
\hline & Systematicity & $5,6,17$ & 316 & 3.35 & 0.75 & 312 & 3.62 & 0.77 \\
\hline & Analyticity & $10,11,15$ & 316 & 3.45 & 0.86 & 312 & 3.79 & 0.77 \\
\hline & Truth-seeking & $13,14,20$ & 316 & 3.46 & 0.77 & 312 & 3.72 & 0.75 \\
\hline & CT Self-confidence & $8,9,19$ & 316 & 3.6 & 0.75 & 312 & 3.85 & 0.78 \\
\hline \multirow{5}{*}{ 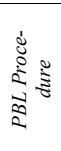 } & Problem Definition & $1,2,4,5$ & 316 & 3.13 & 0.78 & 312 & 3.52 & 0.75 \\
\hline & Hypothesis Proposition & $6,16,17,18$ & 316 & 3.19 & 0.74 & 312 & 3.58 & 0.72 \\
\hline & Independent Inquiry & $3,10,11,15$ & 316 & 3.29 & 0.78 & 312 & 3.75 & 0.73 \\
\hline & Group Negotiation & $7,8,12,13$ & 316 & 3.6 & 0.76 & 312 & 3.84 & 0.74 \\
\hline & Self-reflection & $9,14,19,20$ & 316 & 3.33 & 0.72 & 312 & 3.62 & 0.75 \\
\hline
\end{tabular}

TABLE II.

INDEPENDENT SAMPLE T-TEST OF PRE-Test AND Post-TeST (GENDER)

\begin{tabular}{|c|c|c|c|c|c|c|c|c|c|c|c|c|}
\hline \multirow[t]{3}{*}{ Item Category } & \multicolumn{6}{|c|}{ Pre-test } & \multicolumn{6}{|c|}{ Post-Test } \\
\hline & \multicolumn{2}{|c|}{ Male } & \multicolumn{2}{|c|}{ Female } & \multicolumn{2}{|c|}{ T-test } & \multicolumn{2}{|c|}{ Male } & \multicolumn{2}{|c|}{ Female } & \multicolumn{2}{|c|}{ T-test } \\
\hline & Mean & $S D$ & Mean & $S D$ & $P$ & Sig. & Mean & $S D$ & Mean & $S D$ & $P$ & Sig. \\
\hline In General & 3.28 & 0.36 & 3.36 & 0.36 & -0.53 & 0.60 & 3.66 & 0.25 & 3.72 & 0.29 & $\begin{array}{c}-0.69 \\
\end{array}$ & 0.49 \\
\hline Problem Definition & 3.11 & 0.81 & 3.19 & 0.72 & -0.96 & 0.34 & 3.50 & 0.78 & 3.61 & 0.64 & -0.34 & 0.74 \\
\hline Hypothesis Proposition & 3.18 & 0.76 & 3.22 & 0.71 & -0.54 & 0.59 & 3.60 & 0.71 & 3.53 & 0.75 & 2.03 & $0.04^{*}$ \\
\hline Independent Inquiry & 3.27 & 0.81 & 3.33 & 0.72 & -0.68 & 0.49 & 3.82 & 0.76 & 4.02 & 0.58 & -1.42 & 0.16 \\
\hline Group Negotiation & 3.54 & 0.77 & 3.71 & 0.72 & -2.01 & $0.04^{*}$ & 3.75 & 0.76 & 3.80 & 0.62 & 0.60 & 0.55 \\
\hline Self-reflection & 3.32 & 0.76 & 3.35 & 0.64 & -0.53 & 0.59 & 3.64 & 0.77 & 3.66 & 0.68 & 0.83 & 0.41 \\
\hline Inquisitiveness & 2.96 & 1.03 & 3.15 & 0.89 & -1.49 & 0.14 & 3.58 & 1.01 & 3.74 & 0.78 & -0.92 & 0.36 \\
\hline Open-mindedness & 3.06 & 0.81 & 3.21 & 0.74 & -1.68 & 0.09 & 3.56 & 0.78 & 3.72 & 0.70 & -0.62 & 0.53 \\
\hline Cognitive maturity & 3.03 & 0.80 & 3.13 & 0.74 & -1.18 & 0.24 & 3.47 & 0.78 & 3.41 & 0.70 & 1.57 & 0.12 \\
\hline Systematicity & 3.37 & 0.78 & 3.31 & 0.69 & 0.50 & 0.62 & 3.62 & 0.77 & 3.61 & 0.79 & 1.29 & 0.20 \\
\hline Analyticity & 3.43 & 0.90 & 3.49 & 0.78 & -0.56 & 0.57 & 3.81 & 0.79 & 3.82 & 0.66 & 0.84 & 0.40 \\
\hline Truth-seeking & 3.45 & 0.83 & 3.48 & 0.65 & -0.40 & 0.69 & 3.73 & 0.77 & 3.82 & 0.58 & -0.03 & 0.98 \\
\hline CT Self-confidence & 3.56 & 0.77 & 3.68 & 0.69 & -1.52 & 0.13 & 3.85 & 0.80 & 3.95 & 0.98 & -0.17 & 0.87 \\
\hline
\end{tabular}

${ }^{*} \mathrm{p}<0.05$ (2-tailed)

TABLE III.

CRoss-Test PAIRED-SAMPLE T-TeSt ANALYSIS

\begin{tabular}{|c|c|c|c|c|c|c|c|}
\hline \multirow[t]{2}{*}{ Sample } & \multirow[t]{2}{*}{ Item Category } & \multicolumn{2}{|c|}{ Pre-test } & \multicolumn{2}{|c|}{ Post-test } & \multicolumn{2}{|c|}{ T-test } \\
\hline & & Mean & $S D$ & Mean & $S D$ & $P$ & Sig. \\
\hline \multirow{13}{*}{ Whole } & In General & 3.31 & 0.35 & 3.66 & 0.24 & -3.59 & $0.001^{* *}$ \\
\hline & Problem Definition & 3.13 & 0.78 & 3.52 & 0.75 & -6.23 & $0.000^{*}$ \\
\hline & Hypothesis Proposition & 3.19 & 0.74 & 3.58 & 0.72 & -6.70 & $0.000^{*}$ \\
\hline & Independent Inquiry & 3.29 & 0.78 & 3.75 & 0.73 & -7.61 & $0.000^{*}$ \\
\hline & Group Negotiation & 3.6 & 0.76 & 3.84 & 0.74 & -4.13 & $0.000^{* *}$ \\
\hline & Self-reflection & 3.33 & 0.72 & 3.62 & 0.75 & -5.01 & $0.000^{* *}$ \\
\hline & Inquisitiveness & 3.02 & 0.99 & 3.60 & 0.96 & -7.45 & $0.000^{*}$ \\
\hline & Open-mindedness & 3.11 & 0.79 & 3.59 & 0.77 & -7.72 & $0.000^{*}$ \\
\hline & Cognitive maturity & 3.07 & 0.78 & 3.44 & 0.76 & -6.08 & $0.000^{*}$ \\
\hline & Systematicity & 3.35 & 0.75 & 3.62 & 0.77 & -4.44 & $0.000^{*}$ \\
\hline & Analyticity & 3.45 & 0.86 & 3.79 & 0.77 & -5.21 & $0.000^{*}$ \\
\hline & Truth-seeking & 3.46 & 0.77 & 3.72 & 0.75 & -4.30 & $0.000^{*}$ \\
\hline & CT Self-confidence & 3.6 & 0.75 & 3.85 & 0.78 & -4.10 & $0.000^{*}$ \\
\hline
\end{tabular}

supplements the findings of Wessel and Williams' [15] research which revealed significant effects of $\mathrm{PBL}$ for only three subscales of the CCTDI, namely truth-seeking, $\mathrm{CT}$ self-confidence, and systematicity. In this sense, the findings of the current study were in accordance with that of Tiwari et al. [11] and Kong et al. [12], that is, PBL was able to improve students' $\mathrm{CT}$ in terms of subscales of CCTDI, and further proved that the PBL practice in a College English course improved students' CT disposition in every step of PBL.

\section{CONCLUSIONS}

This research was conducted in an authentic College English in-class context and a WeChat-assisted after-class context, with an Adapted CCTDI as the measurement instrument to collect quantitative data, and with classroom observations of the researcher and written comments of student participants as supplementary qualitative data. The purpose of this current study is to gain a better understanding of the relationship between PBL and the CT disposition of EFL learners.
By undertaking an empirical classroom study, the researcher described the general picture of students' CT disposition before and after their PBL practice, tested the gender differences in respect of the CT subscales and PBL procedures, and found out the impacts of PBL on the CT disposition of EFL learners. The results of this study were as follows:

(1) The mean value of the CT disposition of EFL learners in general was significantly higher in the post-test than that in the pre-test. In specific, student participates were generally better at CT subscales of CT Self-confidence, Analyticity, and Truth-seeking, and performed CT better in PBL steps of Group Negotiation and Independent Inquiry. The students' CT in Independent Inquiry and their Inquisitiveness subscale were obviously improved after the PBL practice.

(2) The mean values of all CT subscales in the post-test were exceedingly and significantly higher than that in the pre-test. 
(3) The mean values of CT disposition in all steps of PBL were exceedingly and significantly higher in the post-test than that in the pre-test.

(4) There was a significant gender difference in Group Negotiation before the PBL practice, but the distinct improvement of male students' CT performance eliminated this significant gender difference after their 9-week PBL experience. The only significant gender difference in Hypothesis Proposition in the post-test was believed not because of the PBL practice or CT itself, but because of the characteristic features and interpersonal interaction habits between males and females in the oriental culture. To be more specific, oriental culture, especially Chinese culture, has been heavily influenced by Confucianism. Confucius and his followers contributed tremendously to the development and maintenance of social, political, and ideological structures of ancient and modern China. However, due to the historical background of feudal ages when Confucian teachings were first taught and venerated, there existed some inevitable limitations in Confucianism, with gender inequality as a case in point. In traditional oriental cultures based on Confucianism, females have been regarded inferior to males, and thus they must show their due respect to male members in their family. Although Chinese women have been struggling hard to subvert this cultural discrimination against them, and they have much bettered their social status in general, it is still a common phenomenon that females have much less talk in either domestic or social contexts. The silence and obedient cooperation of girl students in this PBL practice reflect, to at least some extent, the social reality and female identity in the current Chinese culture. In this very sense, future PBL practices should put more emphasis on the critical thinking of learners regarding cultural discourses, such as gender equality, than merely on linguistic structures.

The conclusions of this study, in answering the research questions, therefore, are (1) that the PBL practice significantly improved EFL students' CT disposition in general, and overtly improved students' CT in Independent Inquiry while maintaining their CT in Group Negotiation; (2) that all of the students' CT subscales, namely Inquisitiveness, Open-mindedness, Cognitive maturity, Systematicity, Analyticity, Truth-seeking, as well as Self-confidence, were significantly improved after the PBL practice; (3) that the EFL learners significantly performed CT better after the PBL practice in all PBL steps, namely Problem Definition, Hypothesis Proposition, Independent Inquiry, Group Negotiation, and Self-reflection; (4) and that the PBL practice eliminated the significant gender difference in Group Negotiation, although there seemed a significant gender difference in Hypothesis Proposition after the PBL practice. In short, the PBL practice in the natural College English teaching context improved the EFL learners' CT disposition in general, as well as in terms of the 7 subscales and in the 5 steps of PBL.

There are four implications of this research for EFL pedagogy. Firstly, PBL in EFL teaching must provide students with opportunities to learn a foreign language and its use by thinking critically while solving a real-world problem. In addition to language knowledge, the EFL learners also learn "how to think" rather than "what to think" [13]. Secondly, compared with the traditional lecture mode of the College English course, PBL approach is more time-consuming and less efficient, therefore it is more practical to integrate $\mathrm{PBL}$ into the existing course, instead of replacing the lecture mode. In this way, PBL approach can be more closely combined with EFL pedagogy via CT. Thirdly, since there is in fact significant gender difference in hypothesis proposition, and groups formed only by girls indeed brought forward less critical and original guessing and proposals according to the classroom observation, it is reasonable to encourage learners to form groups with both male and female members. What's more, the classroom observations also revealed that tutorial groups voluntarily formed by the students performed differently, especially when top students grouped together, and the least abled students were left to form a group. Therefore, it is wise for the tutor to further help learners form heterogeneous groups, with members of different genders, from different backgrounds, and at diverse levels of language proficiency. Finally, the written comments of student participants in this study revealed tremendous uneasy feelings of anxiety, confusion, uncertainty, and even resentment suffered by the EFL learners during their PBL practice. Especially when some students were more apt to the lecture mode and passive learning, they were more dependent and always expected the tutor to provide direct answers and solutions. In this case, an orientation of PBL mode regarding language and its acquisition, as well as the roles of teacher and learner, would be of help to reduce negative affections from the students.

Despite careful planning, the following limitations were identified. The primary limitation of this study exists in the reliance on the Adapted CCTDI and written comments, which were subject to student self-report. Therefore, on the one hand, further investigations involving data other than self-report ones, or a qualitative design of questionnaires with open-ended questions or comment boxes are needed, so that participants have more opportunities to explain why they have chosen certain choice, or to express their own opinion in their own words. On the other hand, the development and validation of a tool to assess CT skills in EFL education using the PBL approach should be an urgent research focus. Another limitation of the current study is the biases in data which should be improved upon in future research. A proper treatment of such kind of systematic errors would improve the validity of the research results. One more limitation is the absence of twogroup research design out of the ethnic consideration of educational research, but findings may be different if other students in the traditional College English course were included in this research. There is indeed a possibility that, in an authentic pedagogic context, other courses the PBL learners in this study took in the same semester also contributed to the improvement of their CT disposition; whereas without a control group, it is less likely to tell for sure to what extent the PBL practice changed the EFL learners' CT. A two-group, pretest-and-posttest research in the future might reveal more details of the changes of students' CT. The last limitation of this study relates to the "Pygmalion effect", the phenomenon whereby the greater the expectation placed upon people, the better they perform. Within the short period of 9 weeks, the student participants were excited about engaging in a research project, and they expected improved CT disposition after the PBL practice as their teachers did. They then attributed traits of the positive expectation with themselves and their $\mathrm{CT}$, and probably overoptimistically reported their CT disposition in the post-test. In this way, the changes of students' CT disposition were somewhat exaggerated by 
PAPER

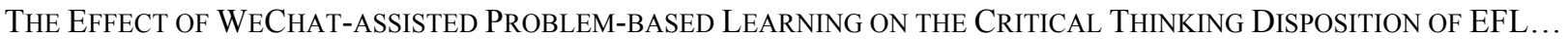

the Pygmalion effect. Therefore, future research should adopt a longitudinal design, which may dramatically eliminate the Pygmalion effect by accustoming the student participants to the PBL context, so that the students gradually enter the desensitization period of the research and behave truly and naturally in the research tests. Such future studies of the effect of PBL on EFL learners' CT disposition and skills may further strengthen the evidence base of effective methods to develop EFL students into critical and life-long language learners.

\section{ACKNOWLEDGMENT}

The author is indebted to the tutors/ instructors and the students who participated in this project.

The author is grateful to her reviewers who generously share with her their academic wisdom and experiences, and offer constructive and thought-provoking comments.

\section{REFERENCES}

[1] N. C. Facione and P. A. Facione, The California Critical Thinking Skills Test and the National League for Nursing Accreditation Requirement in Critical Thinking. Millbrae, California: California Academic Press, 1994, pp. 1-2.

[2] R. Paul and L. Elder, The Miniature Guide to Critical Thinking: Concepts and Tools. Dillon Beach, CA: The Foundation for Critical Thinking, 2001.

[3] D. Margetson, "Why is problem-based learning a challenge?" in The Challenge of Problem-based Learning, $2^{\text {nd }}$ ed., D. Boud and G. Feletti, Eds. London: Kogan Page, 1997, pp. 36-52.

[4] H. S. Barrows and R. M. Tamblyn, Problem-based Learning. New York: Springer, 1980.

[5] D. Boud and G. I. Feletti, "Changing problem-based learning: Introduction to the second edition," in The Challenge of Problembased Learning, 2nd ed., D. Boud and G. I. Feletti, Eds. London: Kogan Page, 1997, pp. 1-14.

[6] X. W. Ding and H. Gu, "A review of PBL models," Journal of Higher Education Research, Vol. 34, pp. 66-69, March 2011.

[7] H. Yuan, B. A. Williams, and L. Fan, "A systematic review of selected evidence on developing nursing students' critical thinking through problem-based learning," Nurse Education Today, Vol.
28, pp. 657-663, June 2008. https://doi.org/10.1016/j.nedt. 2007.12.006

[8] K. J. Oja, "Using problem-based learning in the clinical setting to improve nursing students' critical thinking: an evidence review," Journal of Nursing Education, Vol. 50, pp. 145-151, March 2011. https://doi.org/10.3928/01484834-20101230-10

[9] M. G. Sims, "Efficacy of Problem-Based Learning in promotion of critical thinking in online graduate courses," $\mathrm{Ph}$. D. dissertation, Capella University, Minneapolis, Minnesota, 2008.

[10] W. Shang, "Construction and application of WeChat learning platform in 'Folk Literature' teaching," International Journal of Emerging Technologies in Learning, Vol. 11, pp. 10-15, May 2016. https://doi.org/10.3991/ijet.v11i05.5688

[11] A. Tiwari, P. Lai, M. So, and K. Yuen, "A comparison of the effects of problem-based learning and lecturing on the development of students' critical thinking," Medical Education, Vol. 40, pp. 547-554, June 2006. https://doi.org/10.1111/j.13652929.2006.02481.X

[12] L. N. Kong, B. Qin, Y. Q. Zhou, S. Y. Mou, and H. M. Gao, "The effectiveness of problem-based learning on development of nursing students' critical thinking: A systematic review and metaanalysis," International Journal of Nursing Studies, Vol. 51, pp. 458-469, 2014. https://doi.org/10.1016/j.ijnurstu.2013.06.009

[13] I. Thomas, "Critical thinking, transformative learning, sustainable education, and Problem-based Learning in universities," Journal of Transformative Education, Vol. 7, pp. 245-264, March 2009. https://doi.org/10.1177/1541344610385753

\section{AUTHORS}

Xiao-Wei Ding is with Beijing Science and Technology University, Beijing, 100192, China. She is also a doctoral student at Beijing Foreign Studies University, Beijing, 100089, China. Her research interests include foreign language education and applied linguistics. (e-mail: dingxiaowei@bistu.edu.cn)

This work was supported in part by the grant from Beijing Municipal Commission of Education (Project Code: SM201511232008) and from School of Foreign Studies in Beijing Information Science and Technology University.

Submitted, 10 June 2016. Published as resubmitted by the author on 24 August 2016. 